\title{
DISEÑOS DE ESTUDIOS CLÍNICOS EN ODONTOLOGÍA
}

\section{CLINICAL STUDIES DESIGN IN DENTISTRY}

\author{
Villavicencio-Caparó Ebingen ${ }^{1}$, Alvear-Córdova María ${ }^{1}$, Cuenca-León Katherine ${ }^{1}$, Calderón-Curipoma Mirella ${ }^{1}$, \\ Palacios-Vivar Diego ${ }^{1}$,Alvarado-Cordero Alberto ${ }^{1}$ \\ ${ }^{1}$ Catedrático de Odontología de la Universidad Católica de Cuenca.Ecuador. \\ *evillavicencioc@ucacue.edu.ec
}

\begin{abstract}
Resumen
El objetivo de la sección Tool box, de la Revista Oactiva, tienen la finalidad de brindar una revisión de la literatura, respecto a temas de metodología de la investigación, con el propósito de que los lectores tengan las herramientas necesarias y suficientes para la elaboración de investigaciones útiles para su contexto Latinoamericano.
\end{abstract}

Palabras clave: Diseño de Investigaciones Epidemiológicas,investigación, proyectos de investigación .

\begin{abstract}
The aim of this section is to give a literature review revision about research methodology with the purpose of providing to the readers tools to carry on researches in latin america context.
\end{abstract}

Key words: Epidemiologic Research Design, research,research design..

\section{INTRODUCCIÓN}

El diseño de un estudio, es la estrategia que se emplea para lograr responder a la pregunta de investigación, en ese sentido se tienen perfectamente demarcadas las preguntas que se pueden estudiar en ciencias médicas:

1) Sobre factores de riesgo para contraer la enfermedad.

2) Sobre la prevalencia de la enfermedad.

3) Sobre el pronóstico de la enfermedad.

4) Sobre la efectividad de los métodos terapeúticos para tratar la enfermedad.

5) Sobre la sensibilidad y especificidad de los métodos de diagnóstico para la enfermedad.

En ese sentido existe una relación bi-unívoca entre la pregunta de investigación y el diseño que puede ayudar a contestar la interrogante; así tenemos que para los factores de riesgo se lleva cabo un estudio de caso control, para la prevalencia un diseño transversal, para el pronóstico le corresponde el diseño de cohortes y finalmente para evaluar la efectividad de las terapias se tienen los ensayos clínicos. En los siguientes párrafos se hará una explicación detallada de esta relación.

\section{DISEÑO CASO-CONTROL}

Este diseño responde a la pregunta de investigación ¿Existe relación entre la variable Q con la enfermedad A?. ${ }^{1,2}$ Donde $\mathrm{Q}$ es una variable que se quiere evaluar como factor de riesgo. Se realiza mediante una selección de un grupo de pacientes con la enfermedad presente (casos) y otro grupo con las mismas características, excepto que no tienen la enfermedad(controles). ${ }^{1}$ En ambos grupos se realiza la indagación respecto al factor de riesgo que se espera comprobar que está relacionado con la enfermedad. Este diseño se adecua a las enfermedades de baja prevalencia, debido a que es difícil encontrar los casos, sin embargo, ésta no es la norma para escoger este diseño.

\subsection{AL MOMENTO DE ELEGIR LOS CASOS}

Se debe tener especial cuidado de conocer en qué estadío de la enfermedad están los pacientes, que subtipo de enfermedad tienen y poder medir la progresión de la enfermedad con algún indicador. Por ejemplo, cuando medimos la prevalencia de caries tenemos una medida dicotómica (con caries / sin caries), pero hay diferencia entre alguien que tiene 1 caries activa con otra persona que tiene 14 piezas cariadas, por este motivo se debe plantear un punto de corte, en base a la progresión de la enfermedad, un sub grupo podría ser, los que tienen entre 1 y 3 caries activas y el segundo subgrupo quienes tienen de 4 a más piezas con caries.

\subsection{AL MOMENTO DE ELEGIR LOS CONTROLES}

Se debe tener en cuenta que los pacientes deben provenir de la misma población de los casos. Se puede tener 2 o 3 


\begin{tabular}{|c|c|c|c|}
\hline \multirow{3}{*}{$O \cdot R .=\frac{a * d}{b * c}$} & \multicolumn{2}{|r|}{ Expuesto } & Vo expuestos \\
\hline & Enfermos & 30 (a) & 10 (b) \\
\hline & $\begin{array}{l}\text { Sanos } \\
\text { TOTAL }\end{array}$ & 20 (c) & 40 (d) \\
\hline
\end{tabular}

Tabla 1.

controles por cada caso, sobre todo cuando se tiene un bajo número de casos.

Este diseño es el segundo más económico, debido a que toma datos ya existentes (retrospectivo), por lo tanto el consumo de recursos y de tiempo es bajo. En este diseño un aspecto principal es evitar tener variables confusoras o variables intervinientes, debido a que nos conducirán a tener un sesgo de dimensión incalculable, por lo tanto una conclusión con error tipo I o tipo II. En el ejemplo planteado sobre caries, una variable confusora podría ser si los pacientes consumen algún tipo de medicamento (jarabe), ésto se evita con los criterios de exclusión.

Este diseño tiene un riesgo muy fuerte de sesgo de recuerdo, que consiste en que los pacientes muchas veces no recuerdan si estuvieron expuestos al factor que se está evaluando. Esto se mitiga indagando en la entrevista respecto a estas variables confusoras y posteriormente se puede hacer una corrección matemática en el análisis estadístico. También existe riesgo de sesgo de entrevista, lo cual se mitiga, cegando al participante respecto a la enfermedad y los factores de riesgo que se están evaluando. ${ }^{1}$ Otro límite de este diseño es su baja potencia para lograr conclusiones de causalidad, debido a que no se puede establecer la relación cronológica entre la exposición y la enfermedad. Dado que es un estudio en grupos, también hay una dificultad para calcular la incidencia de la enfermedad.

El estadístico que emplea es el Odds Rattio O.R. que es un valor que va entre cero e infinito. Se interpreta teniendo al número uno como punto de corte, donde los valores inferiores a uno, se interpretan como que existe una asociación inversa, el factor a evaluar resultó ser un factor de protección. Cuando el valor sale uno, significa que no hay ningún tipo de asociación y cuando el valor resulta mayor a uno, se interpreta como que el factor estudiado es un factor de riesgo. En el ejemplo de la tabla 1 se interpreta que los expuestos tienen 6 veces más probabilidad de enfermar que los no expuestos.

\section{DISEÑO DESCRIPTIVO- TRANSVERSAL}

Este diseño responde a la pregunta de investigación ¿Cuánto es la prevalencia de la enfermedad X, en la población estudiada, en el tiempo estudiado $?^{2,3}$ Se realiza este estudio llevando a cabo una sola observación de las unidades de estudio, generalmente se realiza con el objetivo de estimar la prevalencia de una enfermedad, con el propósito de implementar programas de políticas de salud pública. ${ }^{3}$

Es el diseño más económico debido a que el tipo de diagnóstico que se lleva a cabo, necesita ser rápido, de bajo costo y masivo, a expensas de la sensibilidad del método, pues los estudios epidemiológicos sub valúan la enfermedad, dada su característica de estudio poblacional y que no conduce al tratamiento inmediato de los pacientes. Puede dar indicios de factores de riesgo, pero no es el mejor diseño para este fin, dado que los subgrupos que se presentan no tienen el mismo tamaño y esta diferencia de tamaños puede generar un sesgo.

Una característica principal de este estudio es su potencia para generalizar los resultados, debido a que se estudia una muestra y los datos se pueden extrapolar a la población en general. Esta característica se ve garantizada mediante el cálculo del tamaño muestral, realizado con una fórmula que toma en cuenta el $95 \%$ de confianza y el menor error posible, usualmente se admite hasta el $5 \%$. Esta característica tendrá especial importancia al momento de realizar los intervalos de confianza de los resultados obtenidos; mientras mayor sea la muestra el intervalo de confianza será menor, por lo tanto, la estimación de la prevalencia será mejor (estimación puntual).

Un error frecuente en este diseño es tratar de hallar causalidad, asociando variables con la enfermedad. Una fuente importante de sesgo en este tipo de estudios, es la participación de las unidades de estudio, que normalmente acceden a ser investigados aquellos pacientes que tienen la enfermedad y los que están interesados en la misma; generalmente los pacientes indiferentes a la enfermedad que se estudia, rechazan participar en éstos estudios. ${ }^{3}$

El estadístico que se emplea con mayor frecuencia es la prevalencia, que se obtiene de la división de la cantidad de personas enfermas entre la cantidad total de personas examinadas en el estudio.

$$
p=\frac{N r o \_d e \_e n f e r m o s}{\text { Nro_de_total_de_examinados }}
$$

Al momento de planificar el estudio, también se piensa en la posibilidad de publicar los resultados, para esta última parte es muy útil tomar en cuenta las recomendaciones denominadas STrengthening the Reporting of OBservational studies in Epidemiology (STROBE), las cuales son aplicables también para estudios de caso-control y cohortes. ${ }^{4}$

\section{DISEÑO DE COHORTES}

Responde a la pregunta de investigación, ¿Existe asociación entre la variable Q con la enfermedad A?. ${ }^{2,5}$

Para realizar este diseño se establece un grupo poblacional de pacientes sanos y luego se divide en dos subgrupos, en base a alguna exposición que suponemos está asociada a la enfermedad. Es importante mencionar que el investigador no proporciona esta exposición, simplemente es un observador a través del tiempo, no interviene en la designación de expuestos y no expuestos. Otro aspecto importante es que los 


\begin{tabular}{cccc}
\cline { 3 - 4 }$=\frac{\frac{a}{a+c}}{\frac{b}{b+d}}$ & & Expuesto & Vo expuestos \\
\cline { 2 - 4 } & Enfermos & $30(\mathrm{a})$ & $10(\mathrm{~b})$ \\
Sanos & $20(\mathrm{c})$ & 40 (d) \\
TOTAL & & RR=3
\end{tabular}

Tabla 2.

pacientes empiezan todos sanos y a medida que se realiza el estudio van apareciendo los casos de enfermedad. Este diseño es útil en el caso de enfermedades de alta prevalencia, debido a que es fácil encontrar a pacientes que van desarrollando la enfermedad. En contra posición también son útiles en el caso de exposiciones raras. Debido al seguimiento que se hace de los participantes, este estudio usualmente es prospectivo, sin embargo existe la posibilidad de recolectar datos anteriores al estudio, en ese caso se denomina estudio de cohorte retrospectiva-prospectiva. ${ }^{5}$

Este diseño tiene mayor potencia que el de caso-control para demostrar asociación entre el factor de riesgo y la enfermedad, debido a que la enfermedad aparece después de que hemos observado actuar a los factores de riesgo. Sin embargo todavía no se puede concluir causalidad a partir de estos estudios, siempre se necesita hacer el modelo experimental para comprobar causalidad.

El estadístico que emplea es el Riesgo Relativo R.R. que es un valor que va entre cero e infinito. Se interpreta teniendo al número uno como punto de corte, donde los valores inferiores a uno, se interpretan como que existe una asociación inversa, el factor a evaluar resultó ser un factor de protección. Cuando el valor sale uno, significa que no hay ningún tipo de asociación y cuando el valor resulta mayor a uno, se interpreta como que el factor estudiado es un factor de riesgo. En el ejemplo de la tabla 2 se interpreta que los expuestos tienen 3 veces más probabilidad de enfermar que los no expuestos.

\section{DISEÑO EXPERIMENTAL}

Responde a la pregunta: ¿Existe diferencia entre la eficacia del tratamiento X con el tratamiento Y ?. ${ }^{2}$ Se puede responder un sin número de preguntas mediante un ensayo clínico, sin embargo el método implica tener una pregunta principal, que conducirá a plantear el objetivo general, el cálculo del tamaño de la muestra y la redacción de los resultados. ${ }^{6,7}$ Para realizar una pregunta estructurada se utiliza la estrategia PICO.

P de población(grupo de personas en las que se hará el estudio).

I de intervención( tratamiento nuevo).

C de comparado con (segundo tratamiento).

O de outcome o variable resultado.
El diseño experimental, sirve para comprobar la eficacia de una intervención sobre una enfermedad. Se realiza un seguimiento a los pacientes en el tiempo, no es necesario que todos inicien a partir de una misma fecha, sin embargo cada participante debe ser seguido en un punto de tiempo bien definido que se convierte en la línea base o tiempo cero. No se aceptan datos de registros hospitalarios previos, dado que este diseño implica que se tiene que seguir a los pacientes antes de la aplicación de una intervención.

Cabe mencionar también que la mayoría de veces se evalúa una nueva terapia que se compara con la mejor terapia actual estándar, solo si no existe ninguna terapia se compara con un placebo, dependiendo de las implicancias éticas.

Para llevar acabo este tipo de estudio, existe el diseño clásico, el cual consta de 3 momentos, el primero donde las unidades de estudio se separan aleatoriamente en dos grupos uno llamado experimental y otro llamado control. La equivalencia de estos dos grupos al inicio del experimento garantiza que el estudio inició bien. Para saber si esto es así tenemos la primera medición de la variable que se le ha denominado pre test. Es aconsejable que la persona que toma los datos no sepa a que grupo pertenecen las unidades de estudio, con la finalidad de que las mediciones sean lo más objetivas e imparciales. De cumplirse con esta característica, se dice que el estudio es ciego. La finalidad del pre test es saber:

- Si los valores de las variables son equivalentes en ambos grupos.

- Si los valores de las variables nos permiten mejorar mediante una intervención.

- Pronosticar la cantidad de unidades de estudio que pueden mejorar con la intervención.

El segundo momento consiste en aplicar la intervención al grupo experimental y aplicar, un tratamiento convencional o un placebo al grupo control. En este momento se aplica con rigurosidad los procedimientos para la ejecución de la intervención. Es aconsejable que la persona que lleva a cabo estos procedimientos, no sepa de los objetivos del estudio, en ese caso se cumpliría con el concepto del segundo ciego, en los casos que la intervención tiene que ver con síntomas, se recomienda que el doble ciego sea el paciente, de tal forma que sea asignado a un grupo y no sepa si le tocó el grupo experimental o el grupo control, para evitar el efecto placebo.

El tercer momento, consiste en la segunda toma de datos que adopta el nombre de post- test, consiste en medir la variable y valorar si hubo cambio respecto al pre test, si existe cambio la segunda evaluación es para saber si existe diferencia significativa entre el cambio del grupo experimental, comparado con el grupo control. Dado que el observador que midió la variable en el pre test, lo hace también en el post test, se mantiene la calificación de ciego, sin embargo si se desea que exista un tripe ciego, se puede codificar los datos 
y hacer que el tercer involucrado ciego sea el estadístico, de tal manera que solo el investigador principal, sepa cuales son los datos del grupo experimental y del grupo control.

Algunas variaciones de este modelo clásico son: El diseño cruzado, el diseño con abstinencia, el diseño de Solomon, el diseño cuadrado latino y el diseño factorial.

Para dar respuesta a la pregunta de investigación, mediante la comparación del efecto en el grupo experimental y el grupo control, en este diseño se debe utilizar una prueba estadística. Entre las más conocidas tenemos la T de Student y la prueba ANOVA (comparación de promedios) y la prueba Chi cuadrado (comparación de proporciones).

Una fuente de sesgo en este diseño se da al momento de la asignación de los participantes al grupo experimental o al grupo control, esto se mitiga mediante la aleatorización. Esta estrategia ayuda también a controlar las posibles fuentes de sesgo debidas a factores pronósticos conocidos o desconocidos.

Otra fuente de sesgo en este diseño es el abandono del estudio, por parte de un paciente que fue incluido y aleatorizado. Esto repercute en un sesgo que no se puede determinar la magnitud ni la dirección, en tal sentido, la adherencia de los participantes a un ensayo clínico es fundamental. Para evitar estas situaciones los protocolos de los ensayos clínicos incluyen los siguientes datos:

1) Información básica de los pacientes

2) Medidas de adherencia a la intervención del estudio

3) Intervenciones concomitantes

4) Variable (s) de respuesta primaria

5) Variables de respuestas secundarias

6) Otras variables de respuesta pre especificadas

7) Eventos adversos con énfasis en eventos graves y su manejo en caso que se den.

\subsection{FASES DE LOS ENSAYOS CLÍNICOS.}

Generalmente los ensayos de agentes farmaceúticos se han dividido en 4 fases. Los estudios de otro tipo de intervenciones como cambios en el estilo de vida o enfoques quirúrgicos no encajan perfectamente en estas fases, por lo tanto puede ser más fácil clasificarlos como estudios de fase temprana y fase tardía. Para los otros estudios tenemos las siguientes fases:

1. Fase I.- Estudios de tolerancia a fármacos, farmacocinética y farmacodinámica.

1. Fase II.- Estudios terapeúticos exploratorios que examinan los efectos de las dosis y usan biomarcadores como resultado.

1. Fase III.- Estudios de confirmación terapeútica que demuestran el uso clínico y el perfil de seguridad.

1. Fase IV.- Estudios de uso terapeútico que examinan poblaciones amplias o especiales tratando de identificar eventos adversos
Al momento de planificar el estudio, también se piensa en la posibilidad de publicar los resultados, para esta última parte es muy útil tomar en cuenta las recomendaciones denominadas Consolidated Standards of Reporting Trials, CONSORT. ${ }^{8}$

\section{CONCLUSIONES}

Las investigaciones clínicas tienen definidas sus preguntas de investigación y para cada pregunta de investigación existe un diseño de estudio.

Los diseños tienen fortalezas y debilidades, conocer las debilidades y las circunstancias en las que pueden aparecer sesgos, nos permiten plantear estrategias dentro de cada diseño para garantizar la validez de los resultados obtenidos.

\section{Referencias}

1 Levin KA. Study design V. Case-control studies. Evidence Based Dentristry. 2003; 7: p. 83-84.

2 Villavicencio-Caparó E. Lección 1 diseños de investigación [Video] Universidad Católica de Cuenca: Documento Disponible

3 Levin KA. Study design III: Cross-sectional studies. Evidence-Based Dentistry. 2006; 7: p. 24-25.

4 STrengthening the Reporting of OBservational studies in Epidemiology. STROBE statements. [Online].; 2007 [Visitado 2016 Enero 20].Documento Disponible

5 Levin KA. Study design IV: Cohort studies. Evidence Based Dentristry. 2003; 7: p. 51-52.

6 Levin KA. Study design VII. Randomised controlled trials. Evidence Based Dentistry. 2007; 8: p. 22-23.1

7 Friedman L, Furberg C, DeMets D. Fundamentals of clinnical Trials. 4th ed. NY: Springer; 2010.

8 CONSORT Group. CONSORT Transparent reporting of trials. [Online].; 2010.[cited 2016 Enero 22. Documento Disponible
Recibido: 15 de Febrero de 2016

Aceptado: 25 de Febrero de 2016 